\title{
Privatheit ist mehr als Rückzug
}
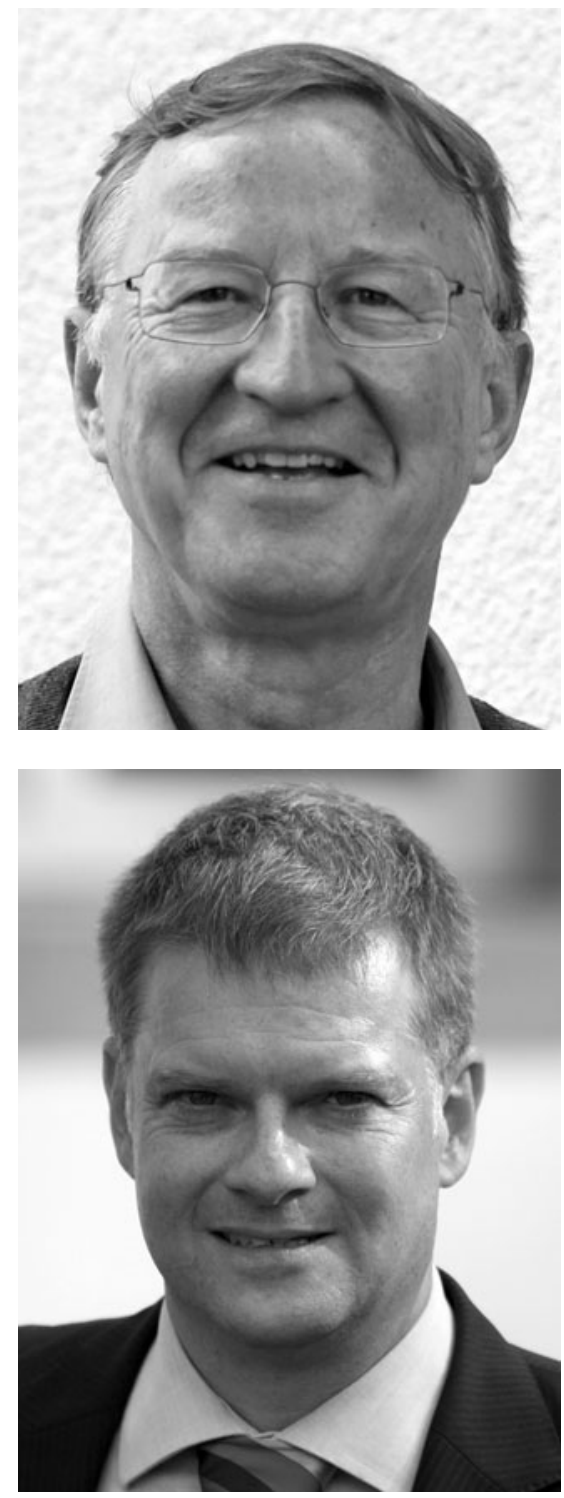

Dass Privatheit als „Recht, in Ruhe gelassen zu werden" ein wesentliches Freiheitsrecht sei, darauf haben Samuel D. Warren und Louis D. Brandeis in ihrem berühmten Beitrag des Harvard Law Review 1890 hingewiesen.' Dieser Schutz des Privaten vor anderen Menschen, insbesondere vor dem Zugriff des Staates, soll auch unter den modernen Kommunikationsbedingungen des Internet und der Mobiltelefonie greifen. Aber hier gerät die Privatheit zunehmend in Gefahr. Selbstdarstellungen in Blogs, Facebook und Twitter, Selbstvermessungen in Sport und Gesundheitsvorsorge (dazu der Beitrag von Ronald Petrlic in diesem Heft) sowie die massenweise Auswertung der Spuren unseres "digitalen Handelns" durch Tracking machen den modernen Menschen gläsern. Davor kann er sich mit Mitteln des Selbstdatenschutzes und einem Mix aus Enthaltsamkeit und aufgeklärtem Einsatz von Abwehrfunktionen zumindest in Grenzen schützen.

Freiheit ist aber nicht nur ein individuelles Genussrecht, sondern für eine demokratische Gesellschaftsordnung schlicht konstitutiv. Demokratie hat die Freiheit nicht nur zum Ziel, sondern auch zur Bedingung: unfreie Menschen können nicht demokratisch handeln. Damit begründete schon 1983 das Bundesverfassungsreicht das Grundrecht der informationellen Selbstbestimmung. Sandra Seubert und Carlos Becker belegen in ihrem Beitrag, warum Freiheit eine Voraussetzung für effektive politische Partizipation ist und jede Asymmetrie von Wissen, von Kommunikations- und Kontrollmacht, bei der wenige vieles über viele wissen, die demokratische Selbstbestimmungspraxis bedroht.

Diese soziale und demokratische Dimension von Privatheit darf man bei der Gestaltung digitaler Kommunikationssysteme nicht aus den Augen verlieren. Damit erweitern sich die Aufgaben der Informatik und der Rechtswissenschaft in diesem Kontext: So rückt der Systemdatenschutz in den Vordergrund, und es wird zur Aufgabe der ganzen Gesellschaft, die Privatheit aller Bürgerinnen und Bürger zu gewährleisten.

Diesen fundamentalen Gedanken der sozialen „Privatheit und Freiheit" behandelte die Konferenz des von der VW-Stiftung geförderten interdisziplinären Projektes „Strukturwandel des Privaten", die im Mai 2015 in Bielefeld stattfand und auf die einige der Beiträge dieses Schwerpunktheftes zurückgehen. Sie reichen von den Möglichkeiten zur rechtlichen Techniksteuerung im luK-Bereich (Philipp Richter) über das Spannungsfeld der Grundrechte zwischen zentralstaatlicher Regulierung und selbstregulierender Aushandlung (Johannes Eichenhofer) bis zu den Möglichkeiten und Grenzen eines technischen Schutzes vor geheimdienstlicher Überwachung aus informatischer Sicht (Stefan Katzenbeisser). Roger Clarke schließlich analysiert in seinem englischsprachigen Beitrag den negativen Einfluss und die gleichwohl positiven Effekte der Informations- und Kommunikationstechnik (IKT) auf Privatheit und Freiheit der Gesellschaft.

Eine zentrale Erkenntnis bleibt:Wir benötigen eine Abkehr von der einseitigen Ausrichtung auf Selbstdatenschutz, die faktisch zu einer Überlastung und Vereinzelung der Bürgerinnen und Bürger geführt hat. Die Betroffenen ertrinken schier in Datenschutzerklärungen und Zustimmungsanforderungen, ohne dass sie dadurch einen Überblick über die weitverzweigte fremde Nutzung ihrer Daten gewinnen. Wenn wir verhindern wollen, dass sich der Schutz der Privatheit und damit die informationelle Selbstbestimmung als Voraussetzung gesellschaftlicher Freiheitsnutzung angesichts überbordender Datenerhebungen und Überwachung in individuellem Fatalismus auflöst, dann müssen wir den Datenschutz in seiner für eine freiheitliche Informationsgesellschaft konstitutiven Dimension begreifen - und als gesamtgesellschaftliche, politische Gestaltungsaufgabe verstehen.

\section{Rüdiger Grimm und Dirk Fox}

1 The Right to Privacy, Samuel D. Warren und Luis D.Brandeis, DuD 10/2012, S. 755 ff. 